\title{
Attention related performance in two cognitively different subgroups of patients with multiple sclerosis
}

\author{
Päivi Kujala, Raija Portin, Antti Revonsuo, Juhani Ruutiainen
}

\begin{abstract}
To evaluate the underlying mechanisms of cognitive decline in multiple sclerosis, two clinically and demographically matched multiple sclerosis groups differing in cognitive status were assessed with attention related tasks. In addition to the attention tests recommended by the Cognitive Function Study Group of the American National Multiple Sclerosis Society, a test of sustained attention was used to evaluate the role of possible fatigue on cognitive performance. The cognitively mildly deteriorated group was slower than the cognitively preserved group and the controls on all tests of attention. The mildly deteriorated group did not, however, consistently differ from the other groups in the error scores of the attention tests. The preserved group exhibited slowness at the end of the visual vigilance test, but no deficits were found on the other attention related tests in this group. It is suggested that dissociable kinds of processing slowness are the origin of the deficits found on the attention tests in the two multiple sclerosis groups. Our preserved group exhibited signs of motor and fatigue related slowness, whereas the mildly deteriorated group also had extensive cognitive slowness. As sensitive indicators of cognitive slowness, attentional tests should be included in evaluation of the cognitive status of patients with multiple sclerosis.
\end{abstract}

(F Neurol Neurosurg Psychiatry 1995;59:77-82)

Masku Neurological Rehabilitation Centre, Finland P Kujala

J Ruutiainen

Department of

Neurology, University of Turku, Finland

R Portin

Centre for Cognitive Neuroscience,

University of Turku,

Finland

A Revonsuo

Correspondence to:

P Kujala, Masku

Neurological Rehabilitation

Centre; PO Box 15

Fin-21251 Masku, Finland

Received 21 July 1994

and in revised form

19 January 1995

Accepted 2 February 1995
Keywords: attention; multiple sclerosis; cognitive impairment; information processing slowness

Disturbances of attention have been used as sensitive indicators of cognitive decline in various diseases. In multiple sclerosis attention has been evaluated with several methods: the paced auditory serial addition test (PASAT), ${ }^{1-5}$ the Stroop test, ${ }^{236-8}$ the Sternberg memory scanning test, ${ }^{1-39}$ and different kinds of vigilance tasks. ${ }^{10-13}$ In some studies, performance of patients with multiple sclerosis on the PASAT has been deficient only on the most demanding conditions ${ }^{1}$ whereas others have found deficiencies also on the easier conditions. ${ }^{3-5}$ On the Stroop test, both attention related deficiencies ${ }^{23}$ and also intact $^{68}$ attention performance have been found. The same holds true with respect to the Sternberg test in which Rao et al ${ }^{239}$ have found patients with multiple sclerosis to be impaired whereas Litvan et al ${ }^{9}$ have found them to be intact. In most multiple sclerosis studies with wide neuropsychological batteries, the digit span has also been used as a measure of short term memory, attention, and concentration. On this test, performance of patients with multiple sclerosis has usually been intact ${ }^{1414-23}$ but deficits have also been found. ${ }^{32}$ Hence the findings on attentional deficits in multiple sclerosis are clearly heterogeneous.

The controversy concerning attentional deficits in multiple sclerosis may be due to the differences in the multiple sclerosis populations studied, especially in the cognitive status of the patients. Cognitive performance of patients with multiple sclerosis has typically been studied by assessing the performance of one cognitively heterogeneous patient group or by classifying the patients into subgroups according to physical disability, disease onset, and duration, ${ }^{16}{ }^{21}$ course of multiple sclerosis, ${ }^{23}$ or certainty of diagnosis. ${ }^{16}$ These subdivision principles are useful when evaluating the relation between cognitive impairment and different disease variables, but are not sensible in studying the underlying mechanisms of cognitive decline in multiple sclerosis. Subdivision according to cognitive status, instead, is a useful method in analysing the mechanisms of cognitive deterioration..$^{25}$

To evaluate the mechanisms of cognitive decline in multiple sclerosis, we assessed two matched multiple sclerosis groups differing only with respect to cognitive status, one with preserved and the other with cognitively mildly deteriorated capacities, with various attentional tests. As well as the attention tests recommended by the Cognitive Function Study Group of the American National Multiple Sclerosis Society, ${ }^{26}$ we used a test of sustained attention to evaluate the role of fatigue on cognitive performance. With this design we attempted to answer the question: What can the attentional deficits of cognitively different patients with multiple sclerosis tell us about the underlying mechanisms of early cognitive decline in multiple sclerosisthat is, in what way and why are the patient groups deficient in cognitive measures?

\section{Methods}

SUBJECTS

The study group consisted of 45 right handed patients with multiple sclerosis who met the 
Table 1 Background variables of the controls and the two patient groups

\begin{tabular}{lccc}
\hline & $\begin{array}{c}\text { Controls } \\
(n=35)\end{array}$ & $\begin{array}{c}\text { Preserved } \\
(n=23)\end{array}$ & $\begin{array}{c}\text { Mildly deteriorated } \\
(n=22)\end{array}$ \\
\hline Male/female & $17 / 18$ & $11 / 12$ & $11 / 11$ \\
Age (y) & $43 \cdot 5(9 \cdot 1)$ & $43 \cdot 3(8 \cdot 7)$ & $43 \cdot 3(7 \cdot 2)$ \\
Formal education (y) & $11 \cdot 3(3 \cdot 2)$ & $11 \cdot 6(3 \cdot 5)$ & $11 \cdot 0(2 \cdot 9)$ \\
Depression score (BDI) ${ }^{33}$ & $2 \cdot 5(3 \cdot 0)^{\star}$ & $7 \cdot 4(6 \cdot 1)$ & $9 \cdot 0(6 \cdot 2)$ \\
Sight & $1 \cdot 6(0 \cdot 3)^{\star}$ & $1 \cdot 4(0 \cdot 3)$ & $1 \cdot 4(0 \cdot 3)$ \\
Colour sight $^{31}$ & $23 \cdot 1(3 \cdot 6)^{\star}$ & $20 \cdot 3(7 \cdot 0)$ & $19 \cdot 0(6 \cdot 2)$ \\
Duration of MS (y) $_{\text {Physical disability (EDSS) }}^{32}$ & & $8 \cdot 7(5 \cdot 9)$ & $8 \cdot 7(6 \cdot 0)$ \\
Course of MS (C-P/R-R/SP) & & $5 \cdot 0(1 \cdot 8)$ & $5 \cdot 5(1 \cdot 3)$ \\
\hline
\end{tabular}

Values (excepting sex and course of multiple sclerosis (MS) are means (SD))

$\star$ The controls differ significantly from the two patient groups. $R-R=$ relapsing-remitting; $\mathrm{C}-\mathrm{P}=$ chronic-progressive; $\mathrm{SP}=$ secondary progressive.

criteria of clinically definite multiple sclerosis according to the definition of Poser et al. ${ }^{27}$ The same patient group was also used in the study of Kujala et al. ${ }^{25}$ Patients with drug or alcohol misuse, psychiatric history, dementia according to DSM III criteria, ${ }^{28}$ severe dysarthria, hearing loss, active phase of disease, or nervous system disorder other than multiple sclerosis were excluded from the study. All patients were assessed with a neuropsychological battery ${ }^{29}{ }^{30}$ for the purpose of selecting patients suitable for either the cognitively preserved or the cognitively mildly deteriorated group. The patients were selected so that the two patient groups were as similar as possible with regard to non-cognitive variables: age, sex, education, depression, sight and colour sight, ${ }^{31}$ duration of multiple sclerosis, physical disability (Kurtzke expanded disability scale) ${ }^{32}$ and course of multiple sclerosis (table 1). The statistical analyses showed that the patient groups did not differ from each other on these variables. All patients were clients of the Masku Neurological Rehabilitation Centre and they were informed about the study during the neurological examination at the beginning of their rehabilitation course.

The control group consisted of 35 right handed healthy subjects with normal hearing. None misused alcohol or drugs, had a psychiatric history, or had a history of brain trauma or disease. The control subjects were staff of the rehabilitation centre and other voluntary participants. The control group was statistically similar to the patient groups with respect to age, sex, and education (table 1). The controls had better sight and colour sight than the patients, but even in the patient groups, visual acuity was normal. All subjects gave their informed consent for participation in this study. The study was approved and carried out in the Masku Neurological Rehabilitation Centre of the Finnish Multiple Sclerosis Society.

\section{DIVISION OF PATIENTS INTO SUBGROUPS}

All subjects were first examined with a neuropsychological battery, which was completed in a single session lasting 60-90 minutes. On the basis of their performance the patients were allocated to one of two groups. The screening tests we used were Finnish versions of the Wechsler adult intelligence scale
(WAIS) ${ }^{34}$ subtests: similarities, digit span, digit symbol, block design and, in addition, the following memory tests: the Benton visual retention test, ${ }^{35} 30$ paired word associates (immediate recall), immediate recall of 20 objects; also naming time of 20 objects was recorded. The patients received deterioration points if their performance on any of the tests was below - 1.5 SD compared with norms based on the control group reported by Portin and Rinne. ${ }^{29}$ If the patient scored below -1.5 SD compared with the norms, he received one deterioration point; if below $-2.0 \mathrm{SD}$, two points; and below $-3.0 \mathrm{SD}$, three points. ${ }^{25} 30$

The classification of patients into the subgroups was made on the basis of deterioration points. Our 45 patients represented the two groups as follows: group 1: cognitively preserved, $0-2$ deterioration points (23 patients); group 2: cognitively mildly deteriorated, 4-12 deterioration points ( 22 patients). The inclusion criterion for the controls was 0-2 deterioration points.

The results of the tests that were used to divide the patients into two groups according to their cognitive status are reported in the study of Kujala et al..$^{25}$ The mildly deteriorated group differed significantly from both the other groups in all screening tests. Individual patients did not, however, receive deterioration points from every screening test. For example, on the WAIS similarities, only one patient received one deterioration point. In addition, the preserved group differed from the controls on two of the tests: on the visuomotor WAIS digit symbol and on the immediate recall of 20 objects. The mean (SD) of the deterioration points was $0.4(0.7)$ for the controls, $0.7(0.8)$ for the preserved group, and $7 \cdot 0(2 \cdot 0)$ for the mildly deteriorated group. The mini mental state examination (MMSE) $^{36}$ was also given to all the subjects. The mildly deteriorated group differed significantly from both the other groups (controls $29.2(0 \cdot 8)$, preserved $29.3(0.9)$, mildly deteriorated $27 \cdot 1(1.9) ; \mathrm{P}<0.001)$. The performance of all patients, apart from one, was, however, within the normal range (24 points or more) on this test.

\section{ATTENTION MEASURES}

Performance on attentional tests was measured in a session in which different neuropsychological tests were carried out, within one week after the screening tests. All tests were given in the same order and at roughly the same time of day for every subject. The PASAT, the Stroop test, the auditory As and trails A tests were recommended by the Cognitive Function Study Group of the American National Multiple Sclerosis Society. ${ }^{26}$ One test of sustained attention was specifically designed for the purpose of studying the effects of fatigue.

Paced auditory serial addition test (PASAT) ${ }^{37} 38$ The PASAT primarily measures attention and processing speed. Subjects are instructed to listen to 61 single digits presented at three 


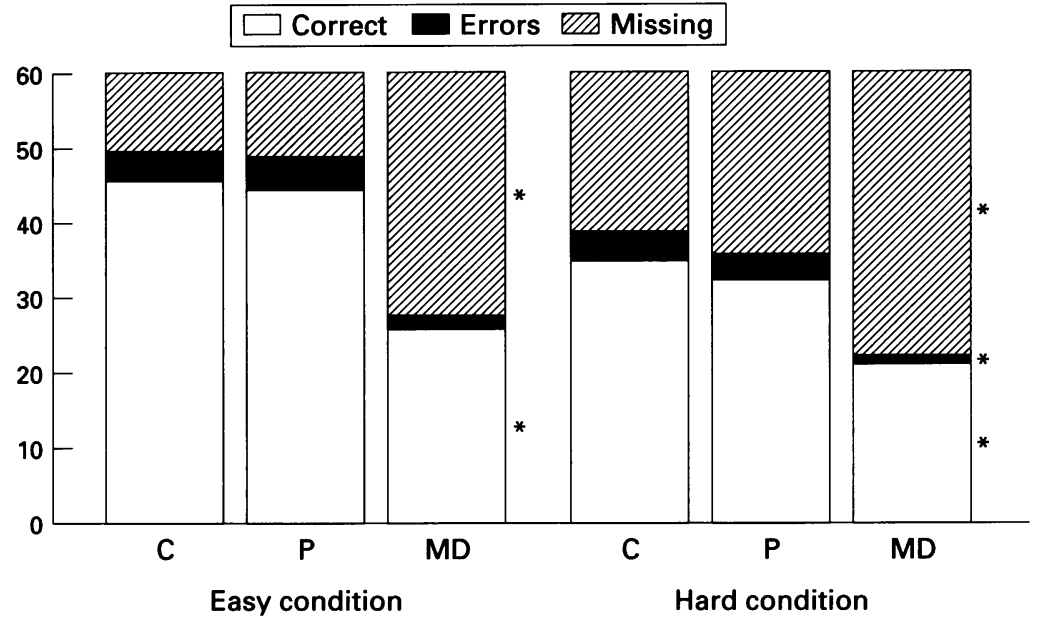

Performance of controls $(C)$ and two patient groups (preserved $(P)$; mildly deterioriated (MD)) on the easy and hard conditions of the PASAT. The means of correct, erroneous, and missing answers (easy condition: correct $P<0 \cdot 001$, errors $P=0 \cdot 12$, missing $P<0.001$; hard condition: correct $P<0.001$, errors $P<0.001$, missing $P<0.001$ ).

*The MD group differs significantly from the other two groups. ensure that the right presentation order is in the subject's mind. On both subtests, the subject is instructed to tap the table with a pencil as soon as the target letter is heard. Furthermore, before both subtests, a practice round is presented. The number of omissions (false negatives) and commissions (false positives) serve as variables in these tasks. Possible effects of fatigue are studied by recording the error rates both of the half and whole tests.

\section{Visual vigilance test}

The visual vigilance test is designed to be a measure of sustained attention. Different letters $(5 \times 5 \mathrm{~mm})$ are presented in the middle of a computer screen (an IBM compatible PC). The presentation time of the letters is $500 \mathrm{~ms}$, and the interval between the letters is $1000 \mathrm{~ms}$. In the 15 minute test, 600 letters are presented, of which 120 are target letters (Y, L). The subject is instructed to keep the preferred hand near the space bar and press it as soon as possible every time he or she sees the letter $\mathrm{Y}$ or the letter $\mathrm{L}$. The subject is told that the computer records, in addition to the correct presses, all omissions and commissions. Furthermore, the time it takes to press the space bar after the target letter has appeared on the screen is recorded. The correct and erroneous presses and reaction times are calculated separately for three five minute periods (0-5 minutes, 5-10 minutes, 10-15 minutes). This is done to study the effects of possible fatigue. The percentage of correct presses, the number of errors, and the group mean of the individual reaction time medians in every three periods serve as variables.

\section{DATA ANALYSES}

Where the two patient groups alone were compared, $t$ tests and $\chi^{2}$ tests were used. Where all three groups were compared, analyses of variance (ANOVAs) and Duncan's multiple comparisons test $(P<0.05)$ were used for parametric variables and KruskalWallis test and Wilcoxon two sample procedures $(\mathrm{P}<0.05)$ for non-parametric variables. Correlation analyses were employed to measure the relation between different variables.

\section{Results}

On the PASAT, the preserved group performed similarly to the controls on all variables measured, whereas the mildly deteriorated group exhibited processing slowness. The mildly deteriorated group produced significantly fewer correct responses and significantly more missing answers than the other two groups both on the easy and on the hard condition of the PASAT (figure). The error rates of the three groups did not differ from each other on the easy condition. On the hard condition, however, the mildly deteriorated group made significantly fewer errors than the other two groups. The mildly deteriorated group gave significantly fewer correct consecutive answers than the other groups (easy condition: controls 20.8 (18.0), subject is instructed to recite the alphabet to 
Table 2 Performance of the controls and the two patient groups on the Stroop test: the means of the naming times, the number of different types of errors, and the interference time $\frac{\text { means of the naming times, the number of different types of errors, and the interference time }}{\text { Controls }}$

\begin{tabular}{lcccc}
\hline & $\begin{array}{c}\text { Controls } \\
\text { Mean (SD) }\end{array}$ & $\begin{array}{c}\text { Preserved } \\
\text { Mean (SD) }\end{array}$ & $\begin{array}{l}\text { Mildly deteriorated } \\
\text { Mean (SD) }\end{array}$ & P value \\
\hline Control condition (colour naming): & & & \\
Naming time (s) & $62.9(11.5)$ & $71.3(14.1)$ & $105.4(25.9)$ & $\mathrm{p}<0.001^{\star}$ \\
Flounderings & $1.0(1.4)$ & $1.0(1.4)$ & $1.0(1.2)$ & $\mathrm{p}=0.96$ \\
Corrected errors & $0.5(1.0)$ & $0.7(1.0)$ & $1.3(1.5)$ & $\mathrm{p}=0.04 \dagger$ \\
Errors & $0.1(0.3)$ & $0.3(0.6)$ & $1.2(1.9)$ & $\mathrm{p}=0.001^{\star}$ \\
Interference condition (colour word naming) & & & \\
Naming time (s) & $104.6(18.6)$ & $112.1(28.7)$ & $182.2(69.5)$ & $\mathrm{p}<0.001^{\star}$ \\
Flounderings & $1.3(1.5)$ & $1.4(1.5)$ & $1.1(1.3)$ & $\mathrm{p}=0.88$ \\
Corrected errors & $0.8(1.3)$ & $0.6(1.0)$ & $1.1(1.4)$ & $\mathrm{p}=0.31$ \\
Errors & $0.5(0.9)$ & $0.5(1.5)$ & $1.4(2.5)$ & $\mathrm{p}=0.08$ \\
Interference time (s) & $41.8(13.2)$ & $40.8(19.9)$ & $76.9(49.9)$ & $\mathrm{p}<0.001^{\star}$ \\
\hline
\end{tabular}

*The mildly deteriorated group differs significantly from the other two groups.

tThe mildly deteriorated group differs significantly from the controls.

preserved $17.6(13 \cdot 8)$, mildly deteriorated 3.3 $(2 \cdot 3), \mathrm{P}<0.001$; hard condition: controls $9 \cdot 2$ $(8 \cdot 3)$, preserved $7 \cdot 3(5 \cdot 6)$, mildly deteriorated $1 \cdot 8(1 \cdot 1), \mathrm{P}<0.001)$.

The results on the Stroop test were of a similar kind to those on the PASAT. The preserved group performed like the controls. By contrast, the mildly deteriorated group exhibited signs of processing slowness, occurring as long naming and interference times (table 2). The numbers of flounderings, corrected errors, and errors were small. The mildly deteriorated group made more corrected errors than the controls and more errors than the other two groups on the control condition; no differences were seen on the interference condition.

As was found on the other two attentional tests of the core battery, ${ }^{26}$ our patients with preserved cognitive capacities did not exhibit signs of deficits either on the auditory As or auditory trails $\mathrm{A}$. On the auditory As test, no commissions were present and only the mildly deteriorated group made omissions. In the mildly deteriorated group, the number of omissions was no larger than in the other two groups on the first half of the test (controls 0.0 , preserved 0.0 , mildly deteriorated $0.1 ; P$ $=0.07$ ) but was larger where the whole test was concerned (controls 0.0 , preserved 0.0 , mildly deteriorated $0.5 ; \mathrm{P}<0.001$ ). On the more difficult auditory trails A test, the deficits of the mildly deteriorated patients were seen throughout the test (omissions and commissions for the first half: controls 0.6 , preserved $0 \cdot 4$, mildly deteriorated $1 \cdot 6 ; \mathrm{P}<$

Table 3 Performance of the controls and the two patient groups on the visual vigilance test: means of the observed targets (\%), the errors rate, and the reaction times

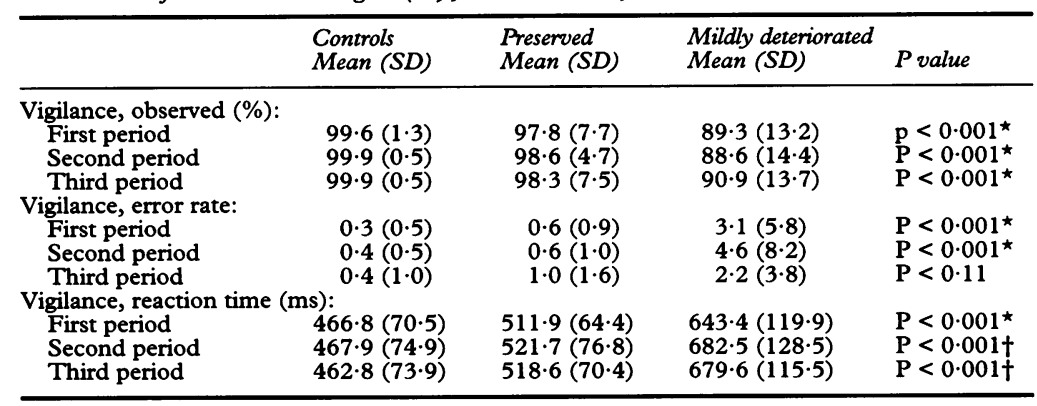

*The mildly deteriorated group differs significantly from the other two groups.

tAll three groups differ significantly from each other.
0.001 ; for the whole test: controls 1.0 , preserved $1 \cdot 2$, mildly deteriorated $3 \cdot 3 ; \mathrm{P}<$ $0.001)$. The errors were mainly omissions, which indicates that the deteriorated patients probably did not have enough time to scan the alphabet in their own memory.

On the visual vigilance test, the controls detected, on average, $99.8 \%$, the preserved group $98.2 \%$, and the mildly deteriorated group $89.5 \%$ of the targets $(P<0.001)$. Although the mildly deteriorated patients differed from the other subjects in this measure, they detected on average 107 of the 120 targets, which indicated that the test was easy. The error rates were low; on the whole test, the controls made on average $1 \cdot 1$, the preserved group $2 \cdot 2$, and the mildly deteriorated group 9.9 commissions out of a total of 480 letters $(P<0.001)$. The mildly deteriorated group made significantly more errors than the other two groups on the first and second period of the test (table 3 ). In the present study, the preserved group exhibited slowness during the last 10 minutes of the test. By contrast, the mildly deteriorated patients were slow throughout the test. All three groups differed from each other in the mean reaction times of the whole test (controls $466 \cdot 2$, preserved 514.5 , mildly deteriorated $669.9 \mathrm{~ms}$; P $<0.001$ ).

The correlations between the main attention variables and depression, sight, and colour sight were non-significant for the whole multiple sclerosis group. In the preserved group, the correlation between colour sight and colour word naming time of the Stroop test was significant $(r=-0.58, P=$ $0.004)$. In the mildly deteriorated group all correlations were non-significant.

\section{Discussion}

In the present study, we attempted to evaluate mechanisms of cognitive decline in multiple sclerosis. We used several tests of attention because evidence exists that attentional deficits may be sensitive indicators of multiple sclerosis related cognitive impairment ${ }^{1011}$ and, furthermore, attentional tests have not been systematically used in previous multiple sclerosis studies. For study purposes, two matched multiple sclerosis groups differing only in cognitive status were assessed and differences in the attentional performance between the patient groups and the controls were evaluated.

Cognitive preservation and different grades of deterioration presumably represent a continuum rather than a dichotomy; our patients were somewhat arbitrarily chosen to exemplify two sections of this continuum-namely, preserved and mildly deteriorated patients. ${ }^{25}$ Our cognitively preserved group differed from the controls in the digit symbol and in the immediate recall of 20 objects. Motor and visual deficits have been found to cause slowness in neuropsychological test performance in patients with multiple sclerosis. ${ }^{7142}$ This kind of slowness probably explained the performance of our preserved patients in the digit 
symbol test. Unfortunately, we did not use a more direct measure of motor performance (for example, tapping). Some of our preserved patients may have had minor cognitive deficits seen as deficient performance in the immediate recall of 20 objects. By contrast, all our mildly deteriorated patients had cognitive deficits and these patients as a group differed from the other two groups in all subtests of the screening battery. The individual patients showed, however, only selective signs of impaired performance. Every patient performed within a normal range on at least two subtests and none of them fulfilled the DSM criteria of dementia. Thus the mildly deteriorated patients can be considered as relatively mildly affected. The subjects were also studied with the MMSE in which the mildly deteriorated group performed significantly worse. The mean score of the mildly deteriorated group was near normal, however, certifying that the MMSE is not sensitive enough to measure the first stages of cognitive impairment. ${ }^{34344}$

The results on the PASAT and on the Stroop test were of a similar kind. The preserved group did not differ from the controls in any of the measures. By contrast, the mildly deteriorated group exhibited processing slowness. On the PASAT, they produced fewer correct and consecutive correct answers than the other subjects, and the number of missing answers was high. On the Stroop test, the mildly deteriorated patients had long naming and interference times. Unexpectedly, the mildly deteriorated patients did not consistently make more errors than the other subjects. Whereas the mildly deteriorated group tended to make fewer errors on the harder subtests of the PASAT and the Stroop test, the increasing cognitive demands were manifested as slowing of processing rates, not as increasing error rates. In previous multiple sclerosis studies with the PASAT ${ }^{1-5}$ and the Stroop test, ${ }^{378}$ the results have been contradictory. This could be due to different test versions, different variables, and, especially, differences in the cognitive status of the patients studied. In this study, patients with mild cognitive deterioration showed extensive processing slowness on attentional measures, whereas the preserved group performed without difficulty.

On the easy tests of auditory and visual sustained attention used in this study, the mildly deteriorated group differed from the other two groups. They exhibited processing slowness by detecting fewer targets and having longer reaction times. On these tests, they also made more errors than the other subjects. Interestingly, the mildly deteriorated group was deficient on the auditory As when the whole test was evaluated but not on the first half. The preserved group, on the other hand, exhibited slowness at the end of the long lasting visual vigilance test. Consequently, both the patient groups exhibited signs of possible fatigue in the tests of sustained attention. In agreement with the fatigue assumption is the fact that the reaction times tended to be short- est in the last period in the controls compared with the first period in the patient groups. The stable course of performance in the controls throughout this test indicates that the test is easy and no learning effects are seen. No analysis of fatigue effects in patients with multiple sclerosis and controls has been reported in previous studies with different versions of attention tests. ${ }^{10-13}$ Our findings concerning fatigue are preliminary and need further support. Therefore, in future studies, fatigue should be systematically evaluated by controlling the course of performance on neuropsychological tests.

One part of the slowness in various attentional measures can be explained by greater physical disability, poorer visual acuity, and a larger number of depression points in the patient groups compared with the controls. The patient groups did not, however, differ from each other in these measures and, consequently, the performance differences between the patient groups cannot be explained by non-cognitive factors. Thus both the patient groups probably have a similar degree of motor slowness. This kind of slowness has also been found in previous multiple sclerosis studies. ${ }^{41}{ }^{42}$ In addition to the motor slowness, signs of fatigue were found in this study. Furthermore, the mildly deteriorated patients had extensive cognitive slowness and this was also seen in our previous study. ${ }^{25}$

The attention related tests of the present study did not measure specific aspects of attention-for example, focused or divided attention-but more general cognitive performance related to attention, working memory, and information processing capacities. With the sensitive attentional tests of the core battery, we found no deficit of any kind in patients with preserved cognitive capacities. By contrast, signs of deficiencies were present on the visual vigilance test. Mild cognitive impairment was, however, clearly seen in the performance on the tests of the core battery. Mild cognitive decline was seen as slow but relatively errorless cognitive processing in patients with multiple sclerosis. On easy and probably automatic tasks, the mildly deteriorated patients made more errors than the other subjects. By contrast, on more complicated tasks, they compensated for their deficits by using slower processing rates. This finding may be relevant in considering the neuropsychological rehabilitation of mild cognitive impairment in multiple sclerosis.

The present study indicates the importance of dividing the cognitively heterogeneous multiple sclerosis group into more homogeneous subgroups to evaluate the possible mechanisms of cognitive decline in multiple sclerosis. Our hypothesis is that at least three types of slowness can alter the performance of patients with multiple sclerosis in the neuropsychological measures: (a) Motor slowness, seen in all patients with physical disability. The effects of this slowness can be manifested in cognitive measures with a main motor component (for example, the digit symbol test, the tapping performance). (b) Fatigue slowness 
seen in many patients with multiple sclerosis presumably regardless of their cognitive capacity. This kind of slowness may affect the performance on tests of sustained attention because of long lasting performance. The origin of fatigue slowness remains to be evaluated in further studies. (c) Cognitive slowness seen in patients with deteriorated cognitive capacities. We propose that this type of slowing may be manifested on most neuropsychological measures. Hence, unlike JennekensSchinkel et al, ${ }^{42}$ we claim that patients with multiple sclerosis may not only have motor but also cognitive slowness. Attentional tests seem to be especially useful in detecting cognitive slowness and mild cognitive decline in multiple sclerosis. To conclude, attentional tests should be systematically used when evaluating the cognitive status of patients with multiple sclerosis.

This study was supported by the Emil Aaltonen Foundation, which is gratefully acknowledged. We thank our patients for their time and interest in this study.

1 Litvan I, Grafman J, Vendrell P, Martinez JM. Slowed information processing in multiple sclerosis. Arch Neurol 1988;45:281-5.

2 Rao SM, Leo GJ, Haughton VM, St Aubin-Faubert P, Bernardin $L$. Correlation of magnetic resonance imaging with neuropsychological testing in multiple sclerosis. Neurology 1989;39:161-6.

3 Rao SM, Leo GJ, Bernardin L, Unverzagt F. Cognitive dysfunction in multiple sclerosis. 1. Frequency, patterns, and prediction. Neurology 1991;41:685-91.

4 De Luca J, Johnson SK, Natelson BH. Information processing efficiency in chronic fatigue syndrome and processing efficiency in chronic fatigue synd
multiple sclerosis. Arch Neurol 1993;50:301-4.

5 Feinstein A, Ron M, Thompson A. A serial study of psychometric and magnetic resonance imaging changes
in multiple sclerosis. Brain 1993;116:569-602.

6 Van den Burg W, van Zomeren AH, Minderhoud JM, Prange AJA, Meijer NSA. Cognitive impairment in patients with multiple sclerosis and mild physical disability. Arch Neurol 1987;44:494-501.

7 Jennekens-Schinkel A, Lanser JBK, van der Velde EA, Sanders EACM. Performances of multiple sclerosis patients in tasks requiring language and visuoconstruction. Assessment of outpatients in quiescent disease stages. F Neurol Sci 1990;95:89-103.

8 van Dijk JG, Jennekens-Schinkel A, Caekebeke JFV, Zwinderman AH. Are event-related potentials in multiple sclerosis indicative of cognitive impairment? Evoked and event-related potentials, psychometric testing and and event-related potentials, psychometric testing and response spee

9 Rao SM, St Aubin-Faubert P, Leo GJ. Information processing speed in patients with multiple sclerosis. f Clin Exp Neuropsychol 1989;11:471-7.

10 Callanan MM, Logsdail SJ, Ron MA, Warrington EK. Cognitive impairment in patients with clinically isolated lesions of the type seen in multiple sclerosis: a psychometric and MRI study. Brain 1989;112:361-74

11 Filley CM, Heaton RK, Nelson LM, Burks JS, Franklin GM. A comparison of dementia in Alzheimer's disease and multiple sclerosis. Arch Neurol 1989;46:157-61.

12 Ron MA, Callanan MM, Warrington EK. Cognitive abnormalities in multiple sclerosis: a psychometric and MRI study. Psychol Med 1991;21:59-68.

13 Feinstein A, Kartsounis LD, Miller DH, Youl BD, Ron MA. Clinically isolated lesions of the type seen in multiple sclerosis: a cognitive, psychiatric, and MRI follow up ple sclerosis: a cognitive, psychiatric, and MR follow

14 Vowels LM. Memory impairment in multiple sclerosis. In: Molloy M, Stanley GV, eds. Brain impairment: proceedings of the third brain impairment workshop. Parkville: Melbourne University Press, 1979.

15 Grafman J, Rao S, Litvan I. Disorders of memory. In: Rao SM, ed. Neurobehavioral aspects of multiple sclerosis. New York, NY: Oxford University Press Inc, 1990.
16 Jennekens-Schinkel A, Laboyrie PM, Lanser JBK, van der Velde EA. Cognition in patients with multiple sclerosis after four years. F Neurol Sci 1989;90:187-201.

17 Caltagirone C, Carlesimo GA, Fadda L, Roncacci S. Cognitive function in multiple sclerosis: a subcortical pattern of neuropsychological impairment? Behavioural Neurology 1991;4:129-41.

18 Klonoff H, Clark C, Oger J, Paty D. Neuropsychological performance in patients with mild multiple sclerosis. f Nerv Ment Dis 1991;179:127-31.

19 Maurelli M, Marchioni E, Cerretano R, Bosone D, Bergamaschi R, Citterio A, et al. Neuropsychological assessment in MS: clinical neurophysiological and neuroradiological relationships. Acta Neurol Scand 1992; 86:124-8.

20 Pozzilli C, Passafiume D, Bernardi S, Pantanto P, Incoccia $\mathrm{C}$, Bastianello $S$, et al. SPECT, MRI and cognitive functions in multiple sclerosis. $\mathcal{F}$ Neurol Neurosurg Psychiatry tions in multiple

21 Grant I, McDonald WI, Trimble MR, Smith E, Reed R. Deficient learning and memory in early and middle phases of multiple sclerosis. I Neurol Neurosurg Psychiatry 1984;47:250-5.

22 Jambor $\mathrm{KL}$. Cognitive functioning in multiple sclerosis. $\mathrm{Br}$ f Psychiatry 1969;115:765-75.

23 Heaton RK, Nelson LM, Thompson DS, Burks JS, Franklin GM. Neuropsychological findings in relapsingremitting and chronic-progressive multiple sclerosis. fournal of Consulting and Clinical Psychology 1985;53:

4 Minden SL, Moes EJ, Orav J, Kaplan E, Reich P. Memory impairment in multiple sclerosis. $\mathcal{F}$ Clin Exp Neuropsychol 1990;12:566-86.

25 Kujala P, Portin R, Revonsuo A, Ruutiainen J. Automatic and controlled information processing in multiple scle117:1115-26.

26 Peyser JM, Rao SM, La Rocca NG, Kaplan E. Guidelines for neuropsychological research in multiple sclerosis. Arch Neurol 1990;47:94-7.

27 Poser CM, Paty DW, Scheinberg L, et al. New diagnostic criteria for multiple sclerosis: guidelines for research protocols. Ann Neurol 1983;13:227-31.

28 American Psychiatric Association. Diagnostic and statistical manual of mental disorders. 3rd ed. Washington, DC: American Psychiatric Association; 1980.

29 Portin R, Rinne UK. Neuropsychological responses of parkinsonian patients to long-term levodopa treatment. In: Rinne UK, Klinger M, Stamm G, eds. Parkinson's disease-current progress, problems and management. disease-current progress, problems and

30 Revonsuo A, Portin R, Koivikko L, Rinne JO, Rinne UK Slowing of information processing in Parkinson's disSlowing of information processing

31 Ishihara S. The series of plates designed as a test for colourblindess. 24 plates edition. Tokyo: Kanehara and Co Ltd, 1987.

32 Kurtzke JF. Rating neurologic impairment in multiple sclerosis: An expanded disability status scale (EDSS). Neurology 1983;33:1444-52.

33 Beck AT, Ward CH, Mendelson M, Mock J, Erbaugh JK An inventory for measuring depression. Arch Gen Psychiatry 1961;4:561-71.

34 Wechsler D. Manual for Wechsler adult intelligence scale. New York: Psychological Corp, 1955.

35 Benton $\mathrm{AL}$. The revised visual retention test. New York: Psychological Corp, 1963.

36 Folstein MF, Folstein SE, McHugh PR. "Mini mental state": a practical method for grading the cognitive state state": a practical method for grading the cognitive state of patients

37 Gronwall DMA. Paced auditory serial addition task: a measure of recovery from concussion. Percept Mot Skills 1977;44:367-73

38 Gronwall D, Wrightson P. Recovery after minor head injury. Lancet 1974;2:1452.

39 Stroop JR. Studies of interference in serial verbal reactions. f Exp Psychol 1935;18:643-62.

40 Lezak MD. Neuropsychological assessment. New York, NY Oxford University Press, 1983.

41 Jennekens-Schinkel AS, Sanders EACM, Lanser JBK, van der Velde EA. Reaction time in ambulant multiple sclerosis patients, part 2 . Influence of task complexity. f Neurol Sci 1988;85:187-96.

42 Jennekens-Schinkel AS, Sanders EACM, Lanser JBK, van der Velde EA. Reaction time in ambulant multiple sclerosis patients, part 1 . Influence of prolonged mental sclerosis patients, part 1. Influence
effort. $\mathcal{F}$ Neurol Sci 1988;85:173-86.

43 Beatty WW, Goodkin DE. Screening for cognitive impairment in multiple sclerosis. Arch Neurol 1990;47: ment in

44 Swirsky-Sacchetti T, Field HL, Mitchell DR, Seward J, Lublin FD, Knobler RL, et al. The sensitivity of the mini-mental state examination in the white matter dementia of multiple sclerosis. F Clin Psychol 1992;48: 